\title{
LeGes
}

Arthur Brunner / Marco Zollinger

\section{Die richterliche Überprüfung von Rechtsverordnungen}

Wenn die Judikative die Vereinbarkeit von Rechtsverordnungen mit dem übergeordneten Recht überprüft, überwacht sie im Kern das Zusammenspiel und Spannungsverhältnis zwischen der Legislative und der Exekutive im Rahmen der Rechtssetzung. Im vorliegenden Beitrag wird zunächst der Frage nachgegangen, in welchem verfahrensrechtlichen Rahmen Rechtsverordnungen richterlich überprüft werden können; sodann wird - entlang der üblichen Auslegungstopoi - dargelegt, wie Gerichte dabei inhaltlich vorgehen (sollten).

Beitragsart: Wissenschaftliche Beiträge

Zitiervorschlag: Arthur Brunner / Marco Zollinger, Die richterliche Überprüfung von Rechtsverordnungen, in: LeGes 32 (2021) 2 


\section{Inhaltsübersicht}

1. Einleitung

2. Der Begriff der Rechtsverordnung

3. Verfahrensrechtliche Aspekte der richterlichen Überprüfung von Rechtsverordnungen

3.1. Prüfungsgefässe

3.1.1. Abstrakte Kontrolle von Rechtsverordnungen

3.1.2. Inzidente Kontrolle von Rechtsverordnungen

3.2. Prüfungsmethodik und Kognition

4. Der Inhalt der gerichtlichen Überprüfung von Rechtsverordnungen

4.1. Das Auslegungselement des Wortlauts

4.2. Das teleologische Auslegungselement

4.3. Das systematische Auslegungselement

4.4. Das gesetzgebungshistorische Auslegungselement

4.4.1. Konsultationsrecht

4.4.2. Genehmigungsvorbehalt

4.4.3. Verordnungsveto

4.4.4. Pflicht der Regierung zur Vorlage des vorgesehenen Verordnungsrechts im Gesetzgebungsprozess

5. Fazit

\section{Einleitung}

[1] Die Rechtsverordnungen von Bund, Kantonen und Gemeinden können von den rechtsanwendenden Behörden - insbesondere den Gerichten - auf ihre Vereinbarkeit mit dem übergeordneten Recht hin überprüft werden. Kontrolliert wird dabei, ob die verschiedenen an der Rechtssetzung beteiligten Akteure sich an den Rahmen der ihnen zukommenden Kompetenzen gehalten haben. Je nachdem, welche Massstäbe die Gerichte zur Anwendung bringen, stärken sie die Legislative oder die Exekutive in ihren Rechtsetzungskompetenzen; die Gerichte entscheiden mithin über Kernfragen der Gewaltenteilung und positionieren sich dabei auch selbst gegenüber den anderen Staatsgewalten. Im vorliegenden Beitrag soll nach kurzen Hinweisen zum Begriff der Rechtsverordnung (Ziff. 2) zunächst beleuchtet werden, in welchen verfahrensrechtlichen «Gefässen» die richterliche Überprüfung von Rechtsverordnungen erfolgt (Ziff. 3). Sodann wird - entlang der üblichen Auslegungstopoi - dargelegt, wie Gerichte dabei inhaltlich vorgehen (sollten) (Ziff. 4).

\section{Der Begriff der Rechtsverordnung}

[2] Bei Verordnungen handelt es sich um generell-abstrakte Rechtsnormen in Gestalt von Erlassen, die in der Normenhierarchie unterhalb von Verfassung und Gesetz stehen. Das Referendum steht gegen sie nicht offen (vgl. Tschannen et al. 2014, § 14 Rz. 1). Verordnungen zeichnen sich daher durch ihre "vereinfachte Erlassform» aus (HäfElin/Haller et al. 2020, Rz. 1851). Ihre Bezeichnung ist uneinheitlich. Verordnungen werden auch als Dekrete, Reglemente, Weisungen, Ordnungen und dergleichen betitelt (vgl. Urteil des BGer 1C_233/2007 vom 14. Februar 2008 E. 4.3). Sie sind von den autonomen Satzungen abzugrenzen, die von Organisationen des öffentlichen Rechts gestützt auf ihre Kompetenz erlassen werden, ihre eigenen Angelegenheiten selbständig zu ordnen (vgl. Häfelin/Müller et al. 2020, Rz. 69 und Rz. 117). Nicht massgebend für das Vorliegen einer Verordnung ist die erlassende Behörde. Sowohl die Exekutive als auch die Legislative und Judikative können Verordnungen erlassen. In letzteren beiden Fällen liegen Parlaments- oder Gerichtsverordnungen vor (vgl. TschanNEN et al. 2014, 
$\S 14$ Rz. 3 ff.). In den meisten Fällen ist allerdings die Exekutive die Verordnungsgeberin (vgl. Uhlmann/Hofstetter 2012, 456 f.). Ob die Regierung selbst oder eine nachgelagerte Verwaltungsbehörde die Verordnung erlässt, ist eine Frage der verfassungs- oder gesetzesrechtlichen Grundlage (vgl. HäFelin/MüLler et al. 2020, Rz. 74 f.).

[3] Abhängig vom Adressatenkreis ist zwischen Verwaltungs- und Rechtsverordnung zu unterscheiden. Rechtsverordnungen richten sich an die Allgemeinheit. Sie regeln das Verhältnis zwischen den Behörden und Privaten und sind infolgedessen amtlich zu publizieren, damit sie rechtswirksam werden (vgl. HäFelin/MülleR et al. 2020, Rz. 78 f.). Verwaltungsverordnungen richten sich demgegenüber im Sinne einer generellen Dienstanweisung von der über- an die untergeordnete Behörde. Sie bezwecken den einheitlichen Vollzug des Verwaltungsrechts oder dienen der Verwaltungs- oder Anstaltsorganisation. Als sogenanntes «Innenrecht» erscheinen sie nicht in der amtlichen Erlasssammlung (Tschannen et al. 2014, § 14 Rz. 10). Da Verwaltungsverordnungen keine Rechten und Pflichten im Aussenverhältnis umschreiben, bilden sie keine Rechtsquellen des Verwaltungsrechts (vgl. BGE 136 V 295 E. 5.7 S. 308; BGE 128 I 167 E. 4.3 S. 171). Entsprechend hat das Bundesgericht ihre Anfechtbarkeit während längerer Zeit generell verneint (vgl. BGE 105 Ib 136 E. 1 S. 139). Die neuere Praxis ist diesbezüglich differenzierter: Soweit Verwaltungsverordnungen auf die Rechtsstellung der Bürgerinnen und Bürger zurückwirken und damit Aussenwirkung entfalten, können sie grundsätzlich zum Gegenstand eines Normenkontrollverfahrens gemacht werden (vgl. BACHMANN 2019, 54 f., m. H.; vgl. zur Überprüfung kantonaler Verwaltungsverordnungen auf dem Wege der abstrakten Normenkontrolle Urteile des BGer 1C_21/2020 vom 23. März 2021 E. 2.2; 2C_613/2015 vom 7. März 2017 E. 2). Wenngleich der Fokus der nachfolgenden Ausführungen auf der richterlichen Überprüfung von Rechtsverordnungen liegt, darf dieser Umstand nicht aus den Augen verloren werden.

[4] Die wichtigste Funktion von Rechtsverordnungen liegt in der Konkretisierung des ihnen übergeordneten Rechts (vgl. Müller 2020, 49 f.; Uhlmann/Hofstetter 2012, 459). Beruht eine Rechtsverordnung direkt auf der Verfassung und konkretisiert sie damit das Verfassungsrecht, handelt es sich um eine selbständige Rechtsverordnung (vgl. Art. 184 Abs. 3 BV; Art. 185 Abs. 3 BV). Unselbständige Rechtsverordnungen stützen sich hingegen auf ein Gesetz. Dient die unselbständige Rechtsverordnung lediglich dem Vollzug dieses Gesetzes, liegt eine Vollziehungsverordnung vor. Vollziehungsverordnungen führen den Gedanken des Gesetzgebers durch Detailvorschriften näher aus oder verdeutlichen das Gesetz. Sie können das Gesetz aber nicht erweitern, abändern oder ergänzen. Insbesondere dürfen sie den Privaten keine neuen Pflichten auferlegen, selbst wenn diese durch den Gesetzeszweck gedeckt wären (vgl. BGE 142 II 451 E. 5.2.7.1 S. 474; BGE 139 II 460 E. 2.2 S. 463; BGE 136 I 29 E. 3.3. S. 33). Die Kompetenz zum Gesetzesvollzug ist regelmässig auf der Verfassungsstufe verankert (vgl. Art. 182 Abs. 2 BV), sodass für den Erlass von Vollziehungsverordnungen grundsätzlich keine gesetzliche Ermächtigung notwendig ist. «Deshalb sind jene Schlusssätze der Gesetze, welche die Behörde zum Vollzuge auffordern, juristisch ohne Bedeutung» (Schneider 1918, 68; vgl. bspw. Art. 32 Abs. 1 Satz 1 des Tierschutzgesetzes vom 16. Dezember 2005 [TSchG; SR 455]). Demgegenüber ergänzt die Verordnungsgeberin mittels (unselbständigen) gesetzesvertretenden Rechtsverordnungen die gesetzliche Regelung, soweit das Gesetz sie hierzu ermächtigt (vgl. Häfelin/Müller et al. 2020, Rz. 96 f.; Tschannen et al. 2014, § 14 Rz. 25 ff.).

[5] Mit der Überprüfung von Rechtsverordnungen überwacht die Judikative im Kern das Zusammenspiel und Spannungsverhältnis zwischen der Legislative und der Exekutive im Rahmen der Rechtssetzung (haupt- oder vorfrageweise; vgl. Ziff. 3.1). Dies entspricht dem Regelfall. Je- 
doch kann gelegentlich auch das Verhältnis zwischen der Legislative und der Judikative zur Diskussion stehen. Begründet eine Gerichtsverordnung Rechte und Pflichten - beispielsweise bei der Bemessung der Gerichtsgebühren oder der Bildung des Spruchkörpers -, liegt ebenfalls eine Rechtsverordnung vor, die der richterlichen Überprüfung zugänglich ist (vgl. bspw. Organisationsreglement des Strafgerichts des Kantons Basel-Stadt vom 16. Dezember 2016 [SG 154.180]; Gebührenverordnung des Obergerichts des Kantons Zürich vom 8. September 2010 [LS 211.11]; vgl. dazu Urteile des BGer 1C_187/2017 und 1C_327/2017 vom 20. März 2018 E. 5 ff.; 2C_110/2008 vom 3. April 2009 E. 2 ff.; vgl. auch HäFelin/Haller et al. 2020, Rz. 1884; Tschannen et al. 2014, § $14 \mathrm{Rz}$. 5). Insofern ist die Rechtsverordnung unter anderem Ausdruck und Ergebnis des Grundsatzes der Gewaltenteilung im jeweiligen Rechtsgebiet.

\section{Verfahrensrechtliche Aspekte der richterlichen Überprüfung von Rechtsverordnungen}

[6] Gemäss dem Stufenbau der Rechtsordnung und dem Rechtsstaatsprinzip (vgl. Art. 5 Abs. 1 BV) muss das Verordnungsrecht den Rahmen des ihm übergeordneten Rechts einhalten. Deshalb hat eine Verordnungsbestimmung des Bundes, die Gesetzes-, Verfassungs- oder Völkerrecht widerspricht, grundsätzlich keinen Bestand. Eine Ausnahme gilt aufgrund des Anwendungsgebots von Art. 190 BV, wenn die fragliche Verordnungsbestimmung letztlich eine verfassungswidrige Gesetzesbestimmung weiterführt oder der Gesetzgeber die Verordnungsgeberin ausdrücklich dazu ermächtigt hat, von der Verfassung abzuweichen (vgl. BGE 136 II 337 E. 5.1 S. 348 f., m.w.H.; Epiney 2015, Rz. 31). Kantonale Verordnungsbestimmungen haben das gesamte Bundesrecht (derogatorische Kraft des Bundesrechts; vgl. Art. 49 Abs. 1 BV), das interkantonale Recht (vgl. Art. 48 Abs. 5 BV) und das übergeordnete kantonale Recht (vgl. Art. 5 Abs. 1 BV) einzuhalten; kommunale Verordnungsbestimmungen zusätzlich das gesamte kantonale Recht und das übergeordnete kommunale Recht (vgl. Kocher 2018, Rz. 486, m.w.H.). Die Unvereinbarkeit von Verordnungsrecht mit übergeordnetem Recht festzustellen, ist insbesondere Sache der (Verwaltungs-)Justiz.

\subsection{Prüfungsgefässe}

[7] Einer gerichtlichen Prüfung zugeführt werden können Verordnungsbestimmungen auf verschiedenen Wegen. Im Verfahren der Erlassanfechtungsbeschwerde können kantonale Verordnungsbestimmungen als solche zum Gegenstand einer gerichtlichen Überprüfung gemacht werden (sog. abstrakte Normenkontrolle). Nicht eingegangen wird vorliegend auf die in den Kantonen teilweise vorzufindenden Verfahren der präventiven Normenkontrolle, denn diese bilden Teil des noch nicht abgeschlossenen Gesetzgebungsverfahrens (vgl. dazu AuER 2016, Rz. 1498 und Rz. 1532 ff.; vgl. aber Ziff. 4.4.1 ff.). Gegen die Verordnungen des Bundes bestehen keine solchen Rechtsmittel: Die abstrakte Normenkontrolle im Rahmen der Beschwerde in öffentlichrechtlichen Angelegenheiten an das Bundesgericht steht aus verfassungsrechtlichen Gründen (vgl. Art. 189 Abs. 4 BV) nur gegen kantonale Erlasse zur Verfügung (vgl. Art. 82 lit. b BGG), und das Bundesverwaltungsgericht beurteilt grundsätzlich nur Verfügungen von Bundesbehörden (vgl. Art. 31 VGG). Bei Gutheissung einer gegen kantonales Verordnungsrecht erhobenen Erlassanfechtungsbeschwerde lautet das Dispositiv des Urteils grundsätzlich auf Aufhebung der als gesetzes-, verfassungs- oder völkerrechtswidrig gerügten Verordnungsbestimmung (vgl. zu 
gewissen Ausnahmefällen Kocher 2018, Rz. 536 f.). Das Gericht wird so - in Anlehnung an Hans Kelsen $(2019,26)$ - zum «negativen Verordnungsgeber». Soweit gegen eine mit dem übergeordneten Recht unvereinbare Verordnungsbestimmung keine Erlassanfechtungsbeschwerde ergriffen wurde oder eine solche nicht zur Verfügung stand, hat dies selbstredend nicht zur Folge, dass diese Norm vor einer gerichtlichen Prüfung ihrer Vereinbarkeit mit dem übergeordneten Recht immunisiert wäre. Vielmehr kann im Rahmen einer späteren Einzelaktkontrolle jederzeit auch der Bestand des im jeweiligen Einzelfall angewendeten Verordnungsrechts infrage gestellt werden (sog. inzidente Normenkontrolle).

\subsubsection{Abstrakte Kontrolle von Rechtsverordnungen}

[8] Kantonale Erlasse - und damit auch kantonale (vgl. statt vieler BGE 145 I 26; Urteile des BGer 2C_110/2008 vom 3. April 2009 E. 1; 1C_312/2007 vom 31. März 2008 E. 1) und kommunale (vgl. Urteil des BGer 2C_110/2017 vom 15. Februar 2017 E. 2.2) Verordnungsbestimmungen - können mit Beschwerde in öffentlich-rechtlichen Angelegenheiten dem Bundesgericht zur (abstrakten) Prüfung unterbreitet werden (vgl. Art. 82 lit. b BGG). Das Bundesrecht verpflichtet die Kantone nicht, diesbezüglich innerkantonale Vorinstanzen zum Bundesgericht vorzusehen (vgl. Doleschal 2019, 34 f.). Soweit die kantonalen Verfassungs- und Gesetzgebungsorgane auf die Einrichtung entsprechender kantonaler Rechtsmittelwege verzichten, ist die direkte Anfechtung beim Bundesgericht möglich (vgl. Art. 87 Abs. 1 BGG).

[9] Allerdings haben doch einige Kantone von der (auch im Bundesgerichtsgesetz vorgesehenen) Möglichkeit Gebrauch gemacht, eine abstrakte Kontrolle von Verordnungsbestimmungen durch ein innerkantonales (Verfassungs-)Gericht vorzusehen. Im Kanton Zürich beurteilt das Verwaltungsgericht auf Beschwerde hin kantonale Erlasse mit Ausnahme der Verfassung und der Gesetze auf ihre Vereinbarkeit mit dem übergeordneten Recht (Art. 79 Abs. 2 KV/ZH [SR 131.211]; Art. 19 Abs. 1 lit. d, Art. 41 Abs. 1 und Art. 42 lit. b Ziff. 3 VRG/ZH [LS 175.2]). Dieselbe Prüfungsbefugnis kommt dem Kantonsgericht Luzern (Art. 188-191 VRG/LU [SRL 40]) und dem Obergericht Schaffhausen (Art. 17 Abs. 1 KV/SH [SR 131.223]; Art. 46 JG/SH [SHR 173.200]) mit Blick auf Rechtssätze verwaltungsrechtlichen Inhalts zu. Ebenso kann das Verwaltungsgericht Aargau kantonale Rechtssätze verwaltungsrechtlichen Inhalts abstrakt überprüfen, wobei sich seine Prüfungsbefugnis auch auf Gesetze erstreckt (§ 70 Abs. 1 VRPG/AG [SAR 271.200]). Die Vereinbarkeit von Verordnungsbestimmungen mit übergeordnetem Recht kann abstrakt ferner vom Obergericht Nidwalden (Art. 59a Abs. 1 und Abs. 2 KV/NW [SR 131.216.2]), dem Appellationsgericht Basel-Stadt (Art. 116 KV/BS [SR 131.222.1]), dem Kantonsgericht Basel-Landschaft (Art. $86 \mathrm{KV} / \mathrm{BL}$ [SR 131.222.2]), dem Verwaltungsgericht Graubünden (Art. 55 Abs. 2 und Abs. 3 KV/GR [SR 131.226]), dem Kantonsgericht Waadt (Art. 131 und Art. 136 KV/VD [SR 131.231]), der Genfer Cour de Justice (Art. 124 lit. a KV/GE [SR 131.234]; Art. 130B LOJ/GE [RS E 2 05]) und dem Kantonsgericht Jura (Art. $104 \mathrm{KV} / J U$ [SR 131.235]) je als Verfassungsgericht überprüft werden. Einige weitere Kantone wie der Kanton Bern (Art. 60 Abs. 1 lit. b Ziff. 1 i.V.m. Art. 2 Abs. 1 lit. b VRPG/BE [BSG 155.21]) kennen eine abstrakte Normenkontrolle bloss für kommunale Erlasse (vgl. mit einer Übersicht DoleschaL, 472 ff.; vgl. auch zu den kantonalen Verfassungsgerichten BolKensteyn 2014, 122 ff.). Soweit ein kantonales Rechtsmittel besteht, ist dieses auszuschöpfen (vgl. Art. 87 Abs. 2 i.V.m. Art. 86 Abs. 1 lit. d BGG), bevor das Bundesgericht angerufen werden kann. Anfechtungsobjekt im bundesgerichtlichen Verfahren bildet dabei sodann der letztinstanzliche kantonale Entscheid. Allerdings kann in den Beschwerdeanträgen neben der 
Aufhebung des angefochtenen Entscheids auch die Aufhebung des (im vorinstanzlichen Verfahren angefochtenen) Erlasses beantragt werden (vgl. zuletzt BGE 145 I 26 E. 1.1 S. 29 f.).

[10] Unabhängig davon, ob eine kantonale Verordnung zunächst innerkantonal angefochten werden musste, oder ob eine beschwerdeführende Person dagegen direkt an das Bundesgericht gelangen konnte, findet der Ausschlusskatalog von Art. 83 BGG keine Anwendung (vgl. Urteil des BGer 2C_380/2016 vom 1. September 2017 E. 1.2 [nicht publ. in BGE 143 II 598]). Was die Beschwerdelegitimation nach Art. 89 Abs. 1 BGG betrifft, muss die beschwerdeführende Person ein innerkantonal zur Verfügung stehendes Rechtsmittel ergriffen haben, bevor sie an das Bundesgericht gelangt (formelle Beschwerde). Mit Blick auf die materielle Beschwerde unterscheiden sich die Anforderungen an die Beschwerdelegitimation nicht von der direkten Anfechtung der Verordnung beim Bundesgericht (vgl. Urteil des BGer 2C_773/2017 vom 13. Mai 2019 E. 1.3): Erforderlich ist, dass die beschwerdeführende Person durch die angefochtene Verordnung aktuell oder virtuell besonders berührt ist und ein schutzwürdiges Interesse an deren Änderung oder Aufhebung hat. Das schutzwürdige Interesse kann rechtlicher oder tatsächlicher Natur sein (vgl. BGE 141 I 36 E. 1.2.3 S. 40; BGE 141 I 78 E. 3.1 S. 81; BGE 138 I 435 E. 1.6 S. 445). Virtuelles Berührtsein setzt voraus, dass die beschwerdeführende Person von der angefochtenen Regelung früher oder später einmal mit einer minimalen Wahrscheinlichkeit unmittelbar betroffen ist (vgl. BGE 139 V 72 E. 2.2 S. 75; BGE 136 I 17 E. 2.1 S. 21; BGE 133 I 206 E. 2.1 S. 210; vgl. zur jüngsten Präzisierung des Erfordernisses der virtuellen Betroffenheit Urteil des BGer 2C_604/2020 vom 9. November 2020 E. 1.3.1-1.3.5).

[11] Die Fristen zur Erhebung einer Erlassanfechtungsbeschwerde knüpfen in aller Regel an die Publikation der betreffenden Verordnung an und sind relativ kurz bemessen (vgl. Art. 101 BGG; $\S 53$ i.V.m. § 22 Abs. 1 und 2 VRG/ZH). Besonderheiten bestehen diesbezüglich in den Kantonen Aargau und Schaffhausen, wo eine jederzeitige Anfechtung möglich ist (vgl. § 70 Abs. 1 VRPG/AG und Art. 51 VRG/SH [SHR 172.200]; mit einer umfassenden Übersicht über die Anfechtungsfristen im interkantonalen Vergleich Doleschal 2019, 710 ff.). Möchte sich die beschwerdeführende Person indes die Möglichkeit offenhalten, gegen den allenfalls nachteiligen kantonalen Entscheid an das Bundesgericht zu gelangen, hat sie im kantonalen Verfahren trotzdem eine 30-tägige Frist ab Inkrafttreten des kantonalen Erlasses einzuhalten (vgl. BGE 137 I 107 E. 1.4 S. 109 ff.). Eine Erlassanfechtungsbeschwerde muss insofern regelmässig zu einem Zeitpunkt erhoben werden, in dem sich noch gar nicht abschätzen lässt, wie die betreffende Bestimmung von den rechtsanwendenden Behörden ausgelegt und angewendet wird. Dies wirkt sich auf den Prüfungsmassstab aus, den die zur Beurteilung berufenen Gerichte bei der abstrakten Überprüfung von Erlassen zur Anwendung bringen: Das Bundesgericht überprüft die Verfassungsmässigkeit eines allgemeinverbindlichen Erlasses im Rahmen der abstrakten Normenkontrolle zwar mit freier Kognition (vgl. Art. 95 BGG), auferlegt sich aber mit Rücksicht auf die verfassungsmässige Kompetenzordnung im föderalistischen Bundesstaat allgemein eine gewisse Zurückhaltung (vgl. BGE 129 I 12 E. 3.2 S. 15). Nach ständiger Rechtsprechung ist massgebend, «ob der betreffenden Norm nach anerkannten Auslegungsregeln ein Sinn zugemessen werden kann, der mit den angerufenen Verfassungs- oder EMRK-Garantien vereinbar ist. Das Bundesgericht hebt eine kantonale Norm nur auf, sofern sie sich jeglicher verfassungs- und konventionskonformen Auslegung entzieht, nicht jedoch, wenn sie einer solchen in vertretbarer Weise zugänglich bleibt» (BGE 140 I 2 E. 4 S. 14; vgl. BGE 138 I 321 E. 2 S. 323). Erscheint eine generell-abstrakte Regelung unter normalen Verhältnissen, wie sie die Verordnungsgeberin voraussetzen durfte, als verfassungsrechtlich zulässig, vermag «die ungewisse Möglichkeit, dass sie 
sich in besonders gelagerten Einzelfällen als verfassungswidrig erweisen könnte, ein Eingreifen des Verfassungsrichters im Stadium der abstrakten Normenkontrolle im Allgemeinen noch nicht zu rechtfertigen». Den Betroffenen verbleibt die Möglichkeit, eine allfällige Verfassungswidrigkeit bei der Anwendung im Einzelfall geltend zu machen (BGE 137 I 77 E. 2 S. 82).

\subsubsection{Inzidente Kontrolle von Rechtsverordnungen}

[12] Stützt sich eine im konkreten Einzelfall ergangene Anordnung (Verfügung) auf eine Verordnungsbestimmung ab, die gegen das übergeordnete Recht verstösst, ist diese Anordnung von den zur Entscheidung berufenen Rechtsmittelinstanzen auf Antrag beschwerdelegitimierter Personen hin aufzuheben. Die Frage, ob die im konkreten Einzelfall angewendete Verordnungsbestimmung mit dem übergeordneten Recht vereinbar ist und Bestand hat (sog. Geltungskontrolle), muss dabei logisch betrachtet beantwortet worden sein, bevor über die richtige Auslegung der als (für sich genommen) rechtswidrig beanstandeten Verordnungsbestimmungen entschieden wird. Denn eine Norm, die keinen Bestand hat, kann nicht (richtig oder falsch) angewendet werden. Entsprechend ist in diesem Zusammenhang von «vorfrageweiser» (oder: inzidenter) Normenkontrolle die Rede. $\mathrm{Zu}$ dieser inzidenten Normenkontrolle sind die Verwaltungsrechtspflegeinstanzen sowohl auf der Ebene der Kantone als auch auf der Ebene des Bundes nicht nur berechtigt, sondern verpflichtet (vgl. Art. 5 Abs. 1 BV; vgl. zur Pflicht kantonaler Rechtsmittelinstanzen, eine als rechtswidrig gerügte kantonale Bestimmung auf ihre Vereinbarkeit mit Bundesrecht zu überprüfen BGE 127 I 185 E. 2 S. 187 f.; Urteil des BGer 2C_747/2010 vom 7. Oktober 2011 E. 4). In der Schweiz existiert mithin ein System diffuser Normenkontrolle, in dem die Kontrolle der Vereinbarkeit einer Norm mit dem übergeordneten Recht grundsätzlich allen rechtsanwendenden Organen übertragen ist (vgl. zu diesem Begriff Auer 2016, Rz. 1499; abzugrenzen ist die diffuse Normenkontrolle danach von Systemen konzentrierter Normenkontrolle, in welchen der jeweilige Verfassungsgeber «die Zuständigkeit, eine Norm auf ihre Übereinstimmung mit höherem Recht zu überprüfen, einem einzigen, speziell dafür eingesetzten Gericht» übertragen hat). Daran ändert nichts, dass in den meisten Fällen letztinstanzlich das Bundesgericht angerufen werden kann und damit doch von einem einzigen Gericht die verbindlichen Massstäbe bestimmt werden.

[13] Die inzidente Normenkontrolle ist kein eigenständiges Rechtsmittel, sondern ein Prüfungsschritt im Rahmen der gerichtlichen Einzelaktkontrolle. Prozessual massgebend für die Anfechtung des betreffenden Einzelakts ist das anwendbare kantonale oder eidgenössische Verfahrensrecht. Im bundesgerichtlichen Verfahren ist die inzidente Normenkontrolle sowohl im Rahmen der ordentlichen Beschwerden (in Zivilsachen, in Strafsachen und in öffentlich-rechtlichen Angelegenheiten) als auch im Rahmen der subsidiären Verfassungsbeschwerde möglich. In diesem Zusammenhang ist einzig darauf hinzuweisen, dass gesteigerte Anforderungen an die Begründung bestehen, wenn vor Bundesgericht die Unvereinbarkeit einer beanstandeten Verordnungsbestimmung mit verfassungsmässigen Rechten gerügt wird (vgl. Art. 106 Abs. 2 BGG).

\subsection{Prüfungsmethodik und Kognition}

[14] Die vorab vorgenommene Unterscheidung zwischen vollziehenden und gesetzesvertretenden Rechtsverordnungen lässt sich nicht immer eindeutig vornehmen und die Übergänge sind regelmässig «fliessend» (Häfelin/Müller et al. 2020, Rz. 94; Nussbaum 2003, 13). Für das Vor- 
gehen bei der richterlichen Überprüfung von Rechtsverordnungen ist die (dogmatische) Differenzierung ohnehin nicht ergiebig, denn ob eine vollziehende oder eine gesetzesvertretende Rechtsverordnung vorliegt, lässt sich nicht ex ante (aufgrund der Selbstdeklaration der Verordnungsgeberin), sondern erst ex post (aufgrund einer materiellen Beurteilung des gerügten Verordnungsrechts) beurteilen. Die Gerichte haben mit anderen Worten in einem ersten Schritt die Gesetzmässigkeit einer Verordnungsbestimmung zu beurteilen, und zwar unabhängig davon, ob die Verordnungsgeberin ihren Erlass als vollziehende oder gesetzesvertretende Rechtsverordnung betrachtet (vgl. BGE 130 I 140 E. 5.3 f. S. 150 ff.). Kommt das Gericht bei dieser Prüfung zum Schluss, dass die Verordnung allein vollziehenden Charakter aufweist, ist deren Vereinbarkeit mit dem vollzogenen Gesetzesrecht automatisch gegeben und eine materielle (Gesetzmässigkeits-)Prüfung der umstrittenen Verordnungsbestimmung erübrigt sich. Geprüft werden muss indes gegebenenfalls die Rüge, die Anwendung der betreffenden Verordnungsbestimmung verletze das dem vollzogenen Gesetz übergeordnete Recht. Während bei kantonalen Verordnungen dieser zweite Prüfungsschritt von Bedeutung ist (vgl. bspw. Urteil des BGer 2P.313/1996 vom 23. März 1999 E. 2 f.), sind bei Vollziehungsverordnungen des Bundes die Erfolgsaussichten einer Verfassungsrüge im Lichte von Art. 190 BV freilich bescheiden.

[15] Kommt das Gericht bei der Prüfung der Gesetzmässigkeit zum Schluss, dass die beanstandete Verordnung Bestimmungen enthält, denen mehr als nur vollziehender Charakter zukommt, stellt sich die Folgefrage, ob die Verordnungsgeberin vom Gesetzgeber für den Erlass dieser Bestimmungen in formeller und materieller Hinsicht hinreichend ermächtigt wurde (vgl. bspw. Urteile des BGer 2C_733/2020 vom 15. März 2021 E. 4; 2C_765/2020 vom 14. Januar 2021 E. 4 f.; 2C_718/2018 vom 27. Mai 2019 E. 4). In diesem Zusammenhang stellen sich Fragen im Hinblick auf die Prüfungsbefugnis der Gerichte: Räumt die bundesgesetzliche Delegationsnorm dem Bundesrat einen sehr weiten Spielraum für die inhaltliche Ausgestaltung der gesetzesvertretenden Rechtsverordnung ein, ist dieser Gestaltungsbereich für das Bundesgericht und die anderen rechtsanwendenden Behörden nach ständiger Rechtsprechung verbindlich (vgl. BGE 144 II 454 E. 3.3 S. 461; BGE 136 II 337 E. 5.1 S. 348). Den Gerichten kann zwar nicht verwehrt sein, eine Prüfung der Verfassungskonformität vorzunehmen, an den gesetzgeberisch gewährten Spielraum zugunsten der Verordnungsgeberin sind sie jedoch aufgrund des Anwendungsgebots für Bundesgesetze gebunden (vgl. Art. 190 BV). Nicht aus verfassungsrechtlichen, sondern aus prozessualen Gründen beschränkt ist die Kognition des Bundesgerichts bei der Überprüfung kantonaler Verordnungen. Die Verletzung des kantonalen Gesetzesrechts kann nur gerügt werden, wenn darin auch eine Verletzung von Bundesrecht zu erblicken ist (vgl. Art. 95 lit. a BGG). Infrage kommt hier namentlich die Rüge, die Verordnungsgeberin habe das Willkürverbot gemäss Art. 9 BV verletzt oder gegen das Gleichbehandlungsgebot im Sinne von Art. 8 Abs. 1 BV verstossen. Eine «freie Überprüfung» des kantonalen Verordnungsrechts kann nicht durch Berufung auf das bundesverfassungsrechtliche Legalitätsprinzip (vgl. Art. 5 Abs. 1 BV) herbeigeführt werden (vgl. Urteile des BGer 2C_33/2021 vom 29. Juni 2021 E. 3.4 .3 i.f.; 2C_700/2018 vom 14. November 2019 E. 2.2; Tschannen et al. 2014, § 19 Rz. 44). Die Prüfungsbefugnis der kantonalen Vorinstanzen des Bundesgerichts - sofern solche bestehen (vgl. Ziff. 3.1.1 hiervor) - geht in diesem Zusammenhang regelmässig weiter. Die kantonalen Gerichte können (und müssen; vgl. Art. 110 BGG; Ziff. 4) die Vereinbarkeit des beanstandeten Verordnungsrechts mit dem übergeordneten Gesetzesrecht frei prüfen.

[16] Was die Frage der verfassungsmässigen Anwendung gesetzesvertretender Rechtsverordnungen angeht, stossen Rechtssuchende bei gesetzesvertretenden Rechtsverordnungen des Bundes 
- wiederum aufgrund des verfassungsrechtlichen Anwendungsgebots für Bundesgesetze (vgl. Art. 190 BV) - jedenfalls dann ins Leere, wenn die gerügte Verfassungsverletzung schon im Gesetz angelegt ist oder der Bundesgesetzgeber dem Bundesrat einen sehr weiten Spielraum für die inhaltliche Ausgestaltung der Verordnung eingeräumt hat und die gerügte Bestimmung sich an diesen Rahmen hält. Dasselbe gilt für kantonale Verordnungen, die auf Bundesgesetzesrecht beruhen (vgl. Urteil des BGer 2C_213/2021 vom 11. Juni 2021 E. 1.5.4). Weitergehende Rügemöglichkeiten werden in diesem Zusammenhang regelmässig nur dort bestehen, wo neben der Verfassungsverletzung auch eine Verletzung der Europäischen Menschenrechtskonvention, allenfalls auch des Freizügigkeitsabkommens mit der Europäischen Union im Raum steht. Keine derartigen Prüfungsschranken bestehen bei kantonalen gesetzesvertretenden Verordnungsbestimmungen, die auf kantonalem Gesetzesrecht beruhen. Hier ist in jedem Fall zu prüfen, ob ihre Anwendung zu einer Verletzung des dem konkretisierten Gesetz übergeordneten Rechts führt.

\section{Der Inhalt der gerichtlichen Überprüfung von Rechtsverordnungen}

[17] Sowohl bei der abstrakten als auch bei der inzidenten Normenkontrolle stellt das Gericht die zu prüfende Norm dem übergeordneten Recht gegenüber. Es bestimmt also mittels Auslegung den Sinn der einander gegenübergestellten Normen - Kocher 2018, Rz. 489 spricht anschaulich von einer «Doppelauslegung» - und entscheidet sodann über die Vereinbarkeit der beanstandeten Verordnungsbestimmung mit dem übergeordneten Recht (vgl. BGE 144 II 454 E. 2 S. 458 f.; Auer 2016, Rz. 1500). Mit Blick auf die Kontrolle kantonaler Rechtsverordnungen ist in Erinnerung zu rufen, dass die letzte kantonale Gerichtsinstanz selbst oder eine vorgängig zuständige andere richterliche Behörde den Sachverhalt frei zu prüfen und das massgebende Recht von Amtes wegen anzuwenden hat (vgl. Art. 110 BGG). Unter formellen Gesichtspunkten als bundesrechtswidrig erweist sich deshalb eine gerichtliche Normenkontrolle, wenn eine Auslegung gänzlich unterbleibt oder sich das kantonale Gericht lediglich auf eine Willkürprüfung beschränkt (vgl. [zwar mit Blick auf kantonales Gesetzesrecht] Urteil des BGer 2C_127/2018 vom 30. April 2019 E. 3.3). Dies gilt selbstredend auch bei überwiegend technischen verordnungsrechtlichen Bestimmungen.

[18] Massgebend für die Auslegung der beanstandeten Verordnungsbestimmung sowie für die Auslegung des übergeordneten Rechts sind grundsätzlich die allgemeinen Auslegungsgrundsätze (vgl. BGE 137 III 217 E. 2.4.1 S. 221 f.), die an dieser Stelle nicht wiederholt zu werden brauchen (vgl. aber den Unterschied zwischen BGE 145 II 270 E. 4.1 S. 271 f. und BGE 144 III 100 E. 5.2 S. 103; vgl. dazu Ziff. 4.1). Soweit es sich beim übergeordneten Recht um Völkerrecht handelt, sind diesbezüglich die Auslegungsgrundsätze der Wiener Vertragsrechtskonvention zu beachten (vgl. Art. 31 ff. VRK [SR 0.111]). Daneben sind bei der gerichtlichen Überprüfung des Bestands von Verordnungsbestimmungen allerdings einige Besonderheiten zu beachten. Diesen Besonderheiten im Zuge der Geltungskontrolle soll nachfolgend nachgespürt werden; sie bilden den eigentlichen Kern dieser Abhandlung. Zunächst sind einige Gedanken zum Auslegungselement des Wortlauts und Wortsinns anzustellen (vgl. Ziff. 4.1). Sodann sind spezifische Fragen zu den Auslegungsgesichtspunkten des Normzwecks und des systematischen Zusammenhangs der betreffenden Bestimmungen zu klären (vgl. Ziff. 4.2 und Ziff. 4.3). Schliesslich ist auf das gesetzgebungshistorische Auslegungselement einzugehen (vgl. Ziff. 4.4). Die folgenden Ausführungen zur Auslegung beziehen sich im Wesentlichen auf die Prüfung der Gesetzmässigkeit von Rechtsverordnungen. Die Prüfung ihrer Verfassungsmässigkeit ist grundsätzlich nicht mehr ein Aspekt 
der Geltungskontrolle, sondern betrifft die Frage, ob die Verordnungsbestimmung im Einzelfall in verfassungskonformer Weise angewendet worden ist.

\subsection{Das Auslegungselement des Wortlauts}

[19] Ausgangspunkt der Auslegung bei der richterlichen Überprüfung von Rechtsverordnungen ist - in Anlehnung an die allgemeinen Regeln - grundsätzlich der Wortlaut der betreffenden Bestimmungen (vgl. BGE 145 II 270 E. 4.1 S. 271). Die Anknüpfung an den Wortlaut ist jedenfalls dann zielführend, wenn der Wortlaut der Gesetzesbestimmung klar ist und mit dem Wortlaut der vollziehenden oder konkretisierenden Verordnungsbestimmungen übereinstimmt. In den Fällen, die den Gerichten zur Prüfung unterbreitet werden, besteht diese grammatikalische Klarheit der einander gegenübergestellten Normen in den allermeisten Fällen nicht.

[20] Schwierigkeiten treten zunächst bei den technischen Verordnungsbestimmungen des Infrastruktur- und Wirtschaftsverwaltungsrechts zutage. Wenn auf der Gesetzesebene beispielsweise lediglich die Rede davon ist, dass der Bundesrat zur Sicherstellung eines behindertengerechten öffentlichen Verkehrssystems Vorschriften über die Gestaltung der Fahrzeuge erlässt (vgl. Art. 15 Abs. 1 lit. c BehiG [SR 151.3]), lässt sich allein aufgrund dieses Wortlauts die Gesetzmässigkeit des daraufhin durch die Verordnungsbestimmung (vgl. Art. 15b Abs. 2 der Verordnung vom 23. November 1983 über Bau und Betrieb der Eisenbahnen [EBV; SR 742.141.1]) vorgenommenen Verweises auf die Verordnung [EU] Nr. 1300/2014 der Kommission vom 18. November 2014 über die technischen Spezifikationen für die Interoperabilität bezüglich der Zugänglichkeit des Eisenbahnsystems der Union für Menschen mit Behinderung und Menschen mit eingeschränkter Mobilität (TSI PRM 2014, ABl. L 356/110 vom 12. Dezember 2014; vgl. Ziff. 6 Anhang 7 EBV) kaum überprüfen. Anzustellen sind vielmehr weitere Überlegungen zum Begriff der Behindertengerechtigkeit, was nur aufgrund des Zwecks von Art. 15 Abs. 1 lit. c BehiG sowie aufgrund der systematischen Stellung dieser Bestimmung - insbesondere auch aufgrund ihres Verhältnisses zu den völkerrechtlichen Verpflichtungen der Schweiz (hier dem Landverkehrsabkommen) - möglich erscheint (vgl. Urteil des BVGer A-359/2018 vom 20. November 2018 E. 7.3.3).

[21] Nicht selten führen Rechtsverordnungen sodann Begriffe ein, die sich auf der Gesetzesstufe nicht finden lassen. Beispielsweise erwähnt das Bundesgesetz vom 20. Juni 2014 über Lebensmittel und Gebrauchsgegenstände (Lebensmittelgesetz, LMG; SR 817.0) den Zwischenhandel, die Zwischenhändlerin oder -händler nicht. In der Verordnung des EDI vom 16. Dezember 2016 betreffend die Informationen über Lebensmittel (LIV; SR 817.022.16) wird der Begriff der Information über Lebensmittel jedoch als jede Information definiert, die ein Lebensmittel betrifft und den Konsumentinnen und Konsumenten, aber auch den Zwischenhändlerinnen und Zwischenhändlern und den Verarbeiterinnen und Verarbeitern durch ein Etikett, durch sonstiges Begleitmaterial oder in anderer Form, einschliesslich über moderne technologische Mittel oder mündlich, zur Verfügung gestellt wird (vgl. Anhang 1 Abs. 1 LIV). Auch bei einer solchen Ausgangslage lässt sich die Gesetzmässigkeit einer Verordnungsbestimmung nicht bloss anhand ihres Wortlauts bestimmen (vgl. Urteil des BGer 2C_733/2020 vom 15. März 2021 E. 4.3.3).

[22] Ausserdem kann der Wortlaut einer gesetzlichen Delegationsnorm nicht mehr ausschlaggebend sein, wenn die Gesetzesbestimmung die Delegationsmaterie mit «namentlich» umschreibt (vgl. Art. 6 Abs. 2 TSchG; Urteil des BGer 2C_765/2020 vom 14. Januar 2021 E. 5.4.2). Gleiches gilt, wenn wie in Art. 15a des Personenbeförderungsgesetzes vom 20. März 2009 (PBG; SR 745.1) zu den Informationspflichten die Wendung «insbesondere [bei Verspätungen oder Ausfällen von 
Verbindungen]» benützt wird. Ob sämtliche in Art. 55b der Verordnung vom 4. November 2009 über die Personenbeförderung (VPB; SR 745.11) vorgesehenen Informationspflichten der im konzessionierten Verkehr tätigen Unternehmen sich auf diese Bestimmung abstützen lassen, kann allein aufgrund des Wortlauts klarerweise nicht beantwortet werden.

[23] Die Unzulänglichkeit des grammatikalischen Auslegungselements führt bei der richterlichen Überprüfung von Rechtsverordnungen bisweilen zu einem Wechsel in der Auslegungsmethodik, sodass die Verordnungsbestimmungen im Lichte der gesetzlichen Delegationsnorm «nach dem Wortlaut, Sinn und Zweck und den ihm zugrunde liegenden Wertungen auf der Basis einer teleologischen Verständnismethode» ausgelegt werden. Massgebend ist in der Folge «die sachlich richtige Entscheidung im normativen Gefüge, ausgerichtet auf ein befriedigendes Ergebnis der ratio legis» (BGE 144 III 100 E. 5.2 S. 103; BGE 141 III 155 E. 4.2 S. 156). Dieser «Paradigmenwechsel» führt die Gerichte unter dem Gesichtspunkt der Gewaltenteilung teilweise in schwierige Entscheidungssituationen: Wenn die Exekutive eine ältere, vom Gesetzgeber nie angetastete Gesetzesbestimmung aufgrund bestimmter Beobachtungen im Gesetzesvollzug um neue Aspekte ergänzt, stellt sich den Gerichten letztlich die Frage, wie weit die Kompetenzen der Exekutive gegenüber dem Gesetzgeber reichen kann.

\subsection{Das teleologische Auslegungselement}

[24] Veranschaulichen lässt sich die vorstehend angedeutete Problematik, wonach der Wortlaut in den Hintergrund der Auslegung rücken kann, anhand von zwei jüngeren Entscheiden des Bundesverwaltungsgerichts, welche den (bewilligungspflichtigen) Personalverleih betrafen. In den beiden Fällen war jeweils zu beurteilen, ob sich der im Jahr 2013 (vgl. AS 2013 5321) erlassene Art. 32 Abs. 2 der Verordnung vom 16. Januar 1991 über die Arbeitsvermittlung und den Arbeitsverleih (AVV; SR 823.111) auf Art. 13 Abs. 1 lit. c des Bundesgesetzes vom 6. Oktober 1989 über die Arbeitsvermittlung und den Arbeitsverleih (AVG; SR 823.11) abstützen lässt. Art. 13 Abs. 1 lit. c AVG sieht vor, dass die Bewilligung für den Personalverleih nur erteilt wird, «wenn der [um die Bewilligung ersuchende] Betrieb kein anderes Gewerbe betreibt, welches die Interessen von Arbeitnehmern oder von Einsatzbetrieben gefährden könnte». Ausdrücklich gestützt auf diese Bestimmung hat der Bundesrat sodann rund 24 Jahre später normiert, dass die Bewilligung verweigert werden kann, wenn der Gesuchsteller Arbeitnehmende aus Einsatzbetrieben, von denen er nicht unabhängig ist, verleihen will (vgl. Art. 32 Abs. 2 AVV). Rein grammatikalisch liegt auf der Hand, dass mit Art. 32 Abs. 2 AVV «eine zusätzliche, [im Verhältnis zu Art. 13 Abs. 1 lit. c AVG] wesentlich andere Voraussetzung für die Bewilligung [...]» - die Unabhängigkeit aufgestellt wurde (Urteil des BVGer B-753/2016 vom 20. September 2017 E. 4.9). Eine grammatikalische Auslegung führt deshalb zum Schluss, dass Art. 32 Abs. 2 AVV sich nicht auf Art. 13 Abs. 1 lit. c AVG abstützen lässt - so auch das Ergebnis des BVGer im Verfahren B-753/2016. Stellt man hingegen den Normzweck von Art. 13 Abs. 1 lit. c AVG in den Vordergrund, ist in Betracht zu ziehen, dass das Personalvermittlungsverhältnis auf einem Interessengegensatz (auch) zwischen dem Personalverleiher und dem verliehenen Arbeitnehmenden beruht. Deshalb können die Interessen der letztgenannten Gruppe gefährdet werden, wenn keine Unabhängigkeit zwischen Personalverleiher und Einsatzbetrieb besteht (vgl. Urteile des BVGer B-270/2018 und B-282/2018 vom 11. Juni 2020 E. 4.4.5). Vor diesem Hintergrund lässt sich, wie dem beim Bundesgericht angefochtenen Urteil des Bundesverwaltungsgerichts vom 11. Juni 2020 in den Ver- 
fahren B-270/2018 und B-282/2018 entnommen werden kann, die Sache durchaus auch anders beurteilen. Ein Entscheid des Bundesgerichts in dieser Angelegenheit steht aus.

[25] Nicht aus den Augen verloren werden darf in Konstellationen wie der vorstehend skizzierten in jedem Fall, dass der Zweck des übergeordneten Gesetzes als solcher nicht zum alleinigen Massstab genommen werden darf. Wie der vom Gesetzgeber anvisierte Gesetzeszweck erfüllt werden soll, ist letztlich eine vorwiegend politische Frage, die zumindest in den Grundzügen vom Gesetzgeber selbst zu entscheiden ist (vgl. Art. $164 \mathrm{BV}$ ). Insofern genügt es nicht, dass sich eine Verordnungsbestimmung auf den Zweck des ihr übergeordneten Gesetzes abstützen kann (im vorstehenden Beispiel: Arbeitnehmerschutz). In jedem Fall ist eine gesetzliche Grundlage in Form einer hinreichend konkreten Gesetzesbestimmung erforderlich. Nur im Rahmen der Auslegung dieser Gesetzesbestimmung - mithin bei der Bestimmung des vom Gesetzgeber für die Verordnungsgeberin abgesteckten Rahmens - kann der teleologische Auslegungstopos eine Rolle spielen. Dies gilt unseres Erachtens nicht nur für das Vollziehungsverordnungsrecht (vgl. BGE 139 II 460 E. 2.2 S. 463, m.w.H), sondern auch für das gesetzesvertretende Verordnungsrecht. Insofern ist konsequent zwischen dem Vorliegen einer hinreichend konkreten, gesetzlichen Delegationsnorm und der Bestimmung des Gesetzeszwecks zu unterscheiden. Als hinreichend konkrete Gesetzesbestimmung erweist sich beispielsweise Art. 6 Abs. 2 TSchG, wonach der Bundesrat Vorschriften über das Halten von Tieren erlässt. Die gestützt auf Art. 6 Abs. 2 TSchG erlassenen, gesetzesvertretenden Vorschriften der Tierschutzverordnung vom 23. April 2008 (TSchV; SR 455.1) sind durch den Gesetzeszweck von Art. 1 TSchG grundsätzlich solange gedeckt, wie sie einen Bezug zur Tierhaltung aufweisen (vgl. [mit Bezug auf Art. 59 Abs. 2 TSchV] Urteil des BGer 2C_765/2020 vom 14. Januar 2021 E. 5.4.2 und E. 5.4.4).

\subsection{Das systematische Auslegungselement}

[26] Aus der systematischen Stellung einer Delegationsnorm im Gesetz lassen sich Hinweise zur Reichweite der delegierten Materie entnehmen. Anknüpfend an das obige Beispiel des Tierschutzgesetzes kann festgestellt werden, dass sich die Delegationsnorm von Art. 6 Abs. 2 TSchG im ersten Abschnitt zum zweiten Kapitel des Tierschutzgesetzes befindet. Dieser Abschnitt betrifft die Tierhaltung als Aspekt des Umgangs mit Tieren (vgl. Urteil des BGer 2C_765/2020 vom 14. Januar 2021 E. 5.4.3). Eine weitere Delegationsnorm findet sich sodann in Art. 10 Abs. 2 TSchG im Abschnitt zur Tierzucht. Aus diesen Beispielen wird ersichtlich, dass die systematische Stellung massgeblich zum Ausdruck bringen kann, welcher Aspekt einer Materie an die Verordnungsgeberin zur weiteren Regelung delegiert wird. Äusserst anschaulich ist in diesem Zusammenhang das Lebensmittelgesetz. Dieses enthält eine Vielzahl von Delegationsnormen, die aufgrund ihrer systematischen Stellung eine gesetzgeberisch bestimmte Reichweite aufweisen (vgl. u.a. Art. 7 Abs. 4 LMG zu den Anforderungen an die Sicherheit von Lebensmittel; Art. 10 Abs. 3 LMG zu den Hygienevorschriften; Art. 13 Abs. 1 LMG zu den Kennzeichnungspflichten; Art. 18 Abs. 4 LMG zum lebensmittelrechtlichen Täuschungsschutz). Nicht umsonst wird in der Rechtssetzungslehre darauf hingewiesen, dass die (äussere) Systematik eines Gesetzes den Regelungen einen bestimmten Sinn gibt und deren Zwecksetzung verständlich macht (vgl. MülLer/UhlmanN 2013, Rz. 203). Die Bedeutung der Systematik ist für die richterliche Überprüfung nicht zu unterschätzen; man darf sich insoweit nicht davon täuschen lassen, dass das systematische Auslegungselement bisweilen im Schatten der gerichtlichen Erwägungen zum Sinn und Zweck einer Bestimmung steht. 
[27] Von der systematischen Stellung der gesetzlichen Delegationsnorm abzugrenzen ist im Grundsatz die Systematik der Rechtsverordnung selbst. Während die Systematik von Vollziehungsverordnungen aufgrund ihres blossen Vollzugscharakters in aller Regel nicht weiter von Belang ist, kann die Systematik einer gesetzesvertretenden Rechtsverordnung aufzeigen, welche delegierte Materie die Verordnungsbestimmung konkretisiert. So hat das Bundesgericht in einer finanzmarktaufsichtsrechtlichen Angelegenheit kürzlich wesentlich auf den Ingress zur überprüften FINMA-Gebührenverordnung abgestellt, in dem der Bundesrat als Verordnungsgeber deklariert hatte, worauf er die Verordnung abstützte (vgl. Urteil des BGer 2C_839/2019 vom 4. Mai 2020 E. 3). Insofern lässt auch die Systematik einer Verordnung Rückschlüsse auf die Gesetzmässigkeit der gesetzesvertretenden Rechtsverordnung zu. Sie bestimmt in gewisser Weise das richterliche «Prüfprogramm». Die «Deklarationen» der Verordnungsgeberin schliessen freilich nicht aus, dass die Gerichte prüfen, ob sich eine Verordnungsbestimmung auch auf andere Gesetzesbestimmungen abstützen lässt. Instruktiv ist in diesem Zusammenhang BGE 144 II 454, in dem das Bundesgericht eine ganze Reihe von Gesetzesbestimmungen (Art. 4 StSG, Art. 84 KEG, Art. 46a RVOG, Art. $4 \mathrm{KHG}$ ) daraufhin überprüfte, ob sie eine hinreichende gesetzliche Grundlage für eine verordnungsrechtliche Regelung über die Kostenüberwälzung im Zusammenhang mit der Abgabe von Jodtabletten auf die Betreiberinnen von Kernkraftwerken bildeten.

[28] Der richterlichen Überprüfung dienlich sind nach dem Gesagten Rechtsverordnungen, deren Systematik sich mit jener des zu konkretisierenden Gesetzes deckt. Der systematischen Konsistenz förderlich ist auch, wenn jede gesetzlich delegierte Regelungsmaterie in eine eigene gesetzesvertretende Rechtsverordnung mündet oder durch die Gliederung der Verordnung zumindest klar zum Ausdruck kommt, was konkretisiert werden soll. In diesem Zusammenhang kann (als positives Beispiel) auf die nicht mehr in Kraft stehende Medizinprodukteverordnung vom 17. Oktober 2001 (MepV; SR 812.213) hingewiesen werden, die unter anderem das Bundesgesetz vom 15. Dezember 2000 über Arzneimittel und Medizinprodukte (Heilmittelgesetz, HMG; SR 812.21) zur Regelungsmaterie der Medizinprodukte konkretisiert hat (vgl. Art. 45 ff. HMG).

\subsection{Das gesetzgebungshistorische Auslegungselement}

[29] Nach der ständigen Rechtsprechung des Bundesgerichts ist die Entstehungsgeschichte einer Norm zwar nicht unmittelbar entscheidend, dient jedoch als Hilfsmittel, um den Sinn einer Norm zu erkennen. Namentlich zur Auslegung neuerer Texte, die noch auf wenig veränderte Umstände und ein kaum gewandeltes Rechtsverständnis treffen, kommt den Materialien eine besondere Bedeutung zu. Dies gilt unseres Erachtens - wie bei den Botschaften zu den Gesetzen gleichermassen für die Erläuterungen zu einer Rechtsverordnung. Im Zusammenhang mit Verordnungen, die der Bundesrat im Zuge der Corona-Pandemie erlassen hat, ist - auch vor dem Hintergrund des besonderen Handlungsdrucks - die These vertreten worden, die Stellungnahmen der in die Ausarbeitung des Normtextes eng involvierten Behörden sei bei Streitfragen im Sinne einer verstärkt authentischen Auslegung besonderes Gewicht beizumessen (vgl. BRUNNER 2020). Um die Berücksichtigung von Materialien und Stellungnahmen als solche soll es nachfolgend jedoch nicht gehen. Im Vordergrund stehen vielmehr die «Interventionsmöglichkeiten», die es der Legislative ermöglichen, während oder nach Abschluss des Gesetzgebungsprozesses auch im Rahmen der Verordnungsgebung Einfluss zu nehmen (Wyss 2020, Rz. 26). Bei der richterlichen Überprüfung von Rechtsverordnungen stellt sich in diesem Zusammenhang die Frage, ob 
eine solche gesetzgeberische Einflussnahme bei der Anwendung und Auslegung der Verordnung zu berücksichtigen ist.

\subsubsection{Konsultationsrecht}

[30] Gemäss Art. 151 Abs. 1 des Bundesgesetzes vom 13. Dezember 2002 über die Bundesversammlung (Parlamentsgesetz, ParlG; SR 171.10) kann die zuständige Kommission verlangen, dass ihr der Entwurf zu einer wichtigen Verordnung des Bundesrats zur Konsultation unterbreitet wird. Ebenfalls bestimmt Art. 22 Abs. 3 ParlG, dass die zuständigen Kommissionen der Bundesversammlung auf Verlangen vor dem Erlass von rechtssetzenden Bestimmungen des Bundesrats konsultiert werden, sofern die Dringlichkeit der Verordnung es zulässt. Aus der Systematik des Parlamentsgesetzes ergibt sich, dass Art. 22 Abs. 3 ParlG das Konsultationsrecht der zuständigen Kommission als Aufgabe der Bundesversammlung definiert, während Art. 151 Abs. 1 ParlG das Verfahren zwischen den Kommissionen und dem Bundesrat regelt (vgl. Odermatt/Tophinke 2014, Rz. 17; Nussbaum 2003, 33). Art. 151 Abs. 3 ParlG verlangt vom Bundesrat zudem, dass er die Bundesversammlung von der Vorbereitung von Verordnungen in Kenntnis setzt.

[31] Im Zuge der richterlichen Überprüfung einer Rechtsverordnung stellt sich die Frage, ob und inwieweit die allenfalls eingereichte Stellungnahme der zuständigen Kommission zum betreffenden Verordnungsentwurf zu berücksichtigen ist. Die Beurteilung der Frage steht vor dem Hintergrund, dass das Konsultationsergebnis für den Bundesrat nicht bindend ist (vgl. Müller 2020, 50; Graf 2014, Rz. 20). Dem Bundesrat steht es demnach zu, an dem in Konsultation gegebenen Verordnungsentwurf trotz einer gegenteiligen Stellungnahme der Kommission festzuhalten. Aus dem Konsultationsverfahren im Sinne von Art. 151 Abs. 1 ParlG können sich daher keine rechtlichen Verpflichtungen für den Bundesrat ergeben. Vielmehr kommt dem Konsultationsergebnis - insbesondere wenn sich die beiden Kommissionen des National- und Ständerats im gleichen Sinn äussern - eine politische Tragweite zu (vgl. Graf 2014, Rz. 20; Nussbaum 2003, 35). Aus rechtlicher Sicht ist das Konsultationsergebnis als eine Empfehlung an den Bundesrat zu verstehen. Überdies steht das Konsultationsrecht nicht der Bundesversammlung, sondern der zuständigen Kommission des National- und Ständerats zu. Es nimmt nicht der Bundesgesetzgeber im eigentlichen Sinn Stellung. Daher lässt sich aus dem Konsultationsergebnis nur bedingt der Wille des Gesetzgebers ableiten. Immerhin kann die zuständige Kommission in ihrer Stellungnahme aber auf die parlamentarische Debatte im Zuge des Gesetzgebungsprozesses im jeweiligen Rat hinweisen. Insofern vermag das Ergebnis der Konsultation den Gerichten als Hilfestellung zur Ergründung des gesetzgeberischen Willens dienen.

[32] Nach der hier vertretenen Auffassung ist das Ergebnis aus dem Konsultationsverfahren gemäss Art. 151 Abs. 1 ParlG bei der Anwendung und Auslegung der betreffenden Rechtsverordnung nicht im Sinne von Materialien eigenständig zu berücksichtigen. Mangels rechtlicher Verbindlichkeit für den Bundesrat ist es nicht angezeigt, der Empfehlung der zuständigen Kommission im gerichtlichen Verfahren nachträglich eine erhöhte oder sogar bindende Wirkung einzuräumen. Den Ratsmitgliedern (vgl. Art. 6 Abs. 1 ParlG) oder der Kommission selbst (vgl. Art. 45 Abs. 1 lit. a ParlG) steht es denn auch frei, auf dem Weg der parlamentarischen Initiative die gesetzliche Delegationsnorm derart anzupassen, dass der Bundesrat zur Änderung der gesetzesvertretenden Rechtsverordnung gezwungen ist. Denkbar wäre auch der Erlass einer der Verordnung widersprechenden Gesetzesbestimmung. Nach dem Dargelegten wäre es daher verfehlt, dem Konsultationsergebnis neben seinem politischen Gehalt im Gerichtsverfahren eine rechtli- 
che Tragweite einzuräumen. Soweit ersichtlich hat das Bundesgericht in seinen Urteilen bisher nicht auf ein Konsultationsergebnis Bezug genommen. Indessen ist unserer Meinung nach nicht ausgeschlossen, dass die Empfehlung der zuständigen Kommission als Orientierungshilfe beigezogen wird, sofern sich daraus Angaben zum Gesetzgebungsprozess und Hinweise auf die Parlamentsdebatte ergeben.

\subsubsection{Genehmigungsvorbehalt}

[33] Einfacher gestaltet sich die Beurteilung, wie es sich mit der (parlamentarischen) Genehmigung einer unselbständigen Rechtsverordnung bei deren Anwendung und Auslegung verhält. Die Genehmigung von Verordnungen des Bundesrats ist in Art. 95 lit. h ParlG im Kontext der Differenzregelung vorgesehen. Der Gesetzgeber kann im Rahmen der gesetzlichen Delegationsnorm eine vorgängige oder nachträgliche Genehmigung der Rechtsverordnung durch die Bundesversammlung vorschreiben. Auch auf der kantonalen Ebene finden sich entsprechende Bestimmungen (vgl. insbesondere Art. 57 Abs. 2 der Walliser Kantonsverfassung [SR 131.232], wonach das Parlament gesetzesvertretende Verordnungen einem Genehmigungsvorbehalt unterwerfen kann; vgl. für eine sachbereichsspezifische Regelung auf kantonaler Ebene Art. 90 Abs. 2 und Art. 91 Abs. 1 des Personalgesetzes des Kantons St. Gallen vom 25. Januar 2011 [sGS 143.1]).

[34] Der Genehmigungsvorbehalt muss im Gesetz ausdrücklich vermerkt werden. Der Genehmigungsbeschluss ist in der Folge (konstitutive) Voraussetzung für die Gültigkeit der Verordnung. Das Inkrafttreten im Falle der vorgängigen Genehmigung oder die Weitergeltung bei einer nachträglichen Genehmigung hängt demnach davon ab, ob die Bundesversammlung bzw. das kantonale Parlament die Verordnung genehmigt (vgl. Sснмid 2018, 170; Nussbaum 2003, 37 f.; vgl. auch Häfelin/Haller et al. 2020, Rz. 1542; Müller/Uhlmann 2013, Rz. 417). Der hierfür bei den Verordnungen des Bundes erforderliche einfache Bundesbeschluss kann nicht einzelne Verordnungsbestimmungen betreffen, sondern bezieht sich auf die Verordnung als Ganzes (vgl. Theler 2014, Rz. 18). Anwendung findet daher in jedem Fall die integral genehmigte Rechtsverordnung. Es besteht daher kein Raum, dem Genehmigungsbeschluss für die Anwendung und Auslegung einer bestimmten Verordnungsbestimmung Hinweise zu entnehmen. Nach der Rechtsprechung des Bundesgerichts hat die Genehmigung denn auch keinen Einfluss auf die richterliche Überprüfbarkeit der Verordnung. In BGE $104 \mathrm{Ib} 412$ hat das Bundesgericht erwogen, dass durch

«die Genehmigung in Form eines einfachen Bundesbeschlusses [...] die Bundesversammlung einer bundesrätlichen Verordnung nichts bei[fügt], was einer Überprüfung durch das Bundesgericht entzogen wäre [...]. [...]. Es kann dahingestellt bleiben, welche rechtliche Bedeutung dem Genehmigungsbeschluss der Bundesversammlung im allgemeinen zukommt. Es genügt im vorliegenden Fall festzustellen, dass ein solcher Beschluss, sofern er in der Form eines einfachen Bundesbeschlusses gefasst worden ist, infolge der gewählten Erlassform vom Bundesgericht überprüft werden kann. Somit muss auch das Zusammenwirken einer bundesrätlichen Verordnung mit einer in Form eines einfachen Bundesbeschlusses erklärten Genehmigung überprüfbar sein» (BGE 104 Ib 412 E. 4c S. 423; vgl. BGE 109 Ib 81 E. 3 S. 86; BGE 106 Ib 182 E. 2 S. 186). 
Demnach dürfen die Gerichte auch bei der Überprüfung einer genehmigten Rechtsverordnung - der erhöhten demokratischen Legitimation unbesehen - jedenfalls zum Schluss gelangen, diese verstosse gegen das Gesetz, auf welchem sie beruht.

\subsubsection{Verordnungsveto}

[35] Vom Genehmigungsvorbehalt abzugrenzen ist das Verordnungsveto. Das Veto ist ein allgemein gehaltenes Einspruchsrecht der Parlamentsmitglieder, das keiner vorgängigen oder ausdrücklichen Verankerung im Rahmen der gesetzlichen Delegationsnorm bedarf (vgl. SснмID 2018, 168 ff.). Das Verordnungsveto konkretisiert das Zusammenspiel von Exekutive und Legislative. Wird es auf der Verfassungsstufe verankert, ist es ein konkretisierendes Element des Grundsatzes der Gewaltenteilung (vgl. SснміD 2018, 178 f.). Der durch sämtliche Kantonsverfassungen garantierte Grundsatz der Gewaltenteilung stellt ein kantonales verfassungsmässiges Recht dar. Steht die Ausübung oder Ausgestaltung eines kantonalen Verordnungsvetos an sich zur Diskussion, prüft das Bundesgericht das Verordnungsveto frei, soweit sich sein Gehalt aus der Kantonsverfassung ergibt (vgl. Art. 95 lit. c BGG; BGE 142 I 26 E. 3.3 S. 30; BGE 138 I 196 E. 4.1 S. 198; BGE 131 I 291 E. 2.1 S. 297; BGE 126 I 180 E. 2a/aa S. 182).

[36] In der üblichen Ausgestaltung des Verordnungsvetos kann eine bestimmte Anzahl von Parlamentsmitgliedern innert einer festgelegten Frist gegen die publizierte Verordnung Einspruch einlegen. Daraufhin hat das Parlament über das Veto zu befinden. Die Verordnung wird aufgehoben, falls die erforderliche (einfache oder qualifizierte) Parlamentsmehrheit dem Einspruch zustimmt (vgl. MüLler 2020, 51). In der Schweiz kennt einzig der Kanton Solothurn ein Verordnungsveto. Gemäss Art. 79 Abs. 3 der Kantonsverfassung des Kantons Solothurn vom 8. Juni 1986 (SR 131.221) können 17 Kantonsräte innert 60 Tagen gegen eine vom Regierungsrat beschlossene Verordnung oder Verordnungsänderung Einspruch einlegen. Wird der Einspruch durch die Mehrheit der anwesenden Kantonsräte bestätigt, ist die Vorlage an den Regierungsrat zurückzuweisen, d. h. die Mitglieder des Parlaments können auf den Verordnungsinhalt keinen unmittelbaren Einfluss nehmen. Vielmehr wird die Verordnung zur Überarbeitung an die Exekutive zurückgewiesen. Immerhin ist der Einspruch «kurz zu begründen» ( $\$ 44$ Abs. 2 des Kantonsratsgesetzes des Kantons Solothurn vom 24. September 1989 [BSG 121.1]).

[37] Ergibt sich aus der Begründung des Einspruchs, weshalb dieser erhoben wurde, oder aus der nachfolgenden parlamentarischen Debatte, weswegen die Verordnung aufgehoben wurde, lassen sich daraus Erkenntnisse über den gesetzgeberischen Willen gewinnen. Bei der richterlichen Überprüfung der Rechtsverordnung stellen sich deshalb folgende Fragen: Ist eine im Rahmen eines (kantonalen) Verordnungsvetos aufgehobene Verordnung bei der Anwendung und Auslegung der neu erarbeiteten, nunmehr in Kraft stehenden Rechtsverordnungen zu berücksichtigen? Wie verhält es sich mit der Begründung des Einspruchs durch das Parlamentsmitglied? Im Gegensatz zum Konsultationsrecht, das lediglich der zuständigen Kommission zukommt, steht die Kompetenz zur Aufhebung einer Verordnung dem Parlament zu. Letzteres hat bereits das entsprechende Gesetz samt gesetzlicher Delegationsnorm beschlossen. Der Beschluss des Parlaments wirkt kassatorisch und ist für die Verordnungsgeberin bindend (vgl. Schмid 2018, 166). Folglich bringt das Parlament mit der Aufhebung (wirksam) zum Ausdruck, dass die Verordnung (in gewissen Aspekten) nicht dem gesetzgeberisch Gewollten entspricht. Diese Willensäusserung des Parlaments ist unserer Auffassung nach von den Gerichten zu berücksichtigen. Es wäre fragwürdig, wenn ein Gericht bei der Auslegung der neuen Rechtsverordnung zu einem Ergebnis gelangt, das im 
Wesentlichen der Regelung entspricht, die das (kantonale) Parlament durch die Aufhebung der vormaligen Verordnung verhindern wollte. Anders verhält es sich, wenn der erhobene Einspruch im Parlament keine Mehrheit findet. Diesfalls sollte der begründete Einspruch von den Gerichten nicht beachtet werden. In der bundesgerichtlichen Rechtsprechung finden sich keine Urteile, die in entscheidrelevanter Weise auf ein Verordnungsveto Bezug nehmen (vgl. aber Urteil des BGer 2P.44/1993 vom 17. März 1995 E. 2.a).

\subsubsection{Pflicht der Regierung zur Vorlage des vorgesehenen Verordnungsrechts im Gesetzgebungsprozess}

[38] Im Jahr 2018 hat der St. Galler Kantonsrat die Motion 42.18.07 «Einbezug des Kantonsrates beim Verordnungsrecht» gutgeheissen. Damit wurde der Regierungsrat eingeladen, dem Kantonsrat den Entwurf eines Nachtrags zum Staatsverwaltungsgesetz (StVG/SG; sGS 140.1) zu unterbreiten, der vorsieht, dass die Regierung dem Kantonsrat mit der Vorlage für einen Gesetzeserlass im Rahmen der Botschaft auch die Grundzüge des angedachten zugehörigen Verordnungsrechts unterbreitet, wenn die entsprechende Verordnung eine politische Aussenwirkung hat oder von Amtes wegen in der Gesetzessammlung veröffentlicht wird. Damit soll gesetzlich vorgeschrieben werden, was die St. Galler Regierung in jüngerer Vergangenheit (z.B. im Rahmen der Totalrevision des Planungs- und Baugesetzes [sGS 731.1]) teilweise bereits freiwillig praktiziert hat. Am 23. Mai 2021 hat die Staatskanzlei des Kantons St. Gallen einen Bericht und einen Entwurf für eine entsprechende Gesetzesanpassung vorgelegt (RRB 2021/205).

[39] Der Entwurf sieht in einem neu zu erlassenden Art. 5 Abs. $1^{\text {bis }}$ StVG/SG vor, dass die Regierung dem Kantonsrat bei Entwürfen mit Gesetzesrang im Rahmen der Botschaft auch die Grundzüge des angedachten zugehörigen Verordnungsrechts unterbreitet, wenn die Verordnung von erheblicher Bedeutung ist (vgl. auch die entsprechende, per 1. April 2021 in Kraft getretene Regelung in Art. 64a des Bündner Gesetzes vom 8. Dezember 2005 über den Grossen Rat [GRG; BR 170.100]). Dies wird es dem Kantonsrat ermöglichen, bei der Beratung der Gesetzesvorlage das geplante Verordnungsrecht zu würdigen, die Gesetzesvorlage entsprechend anzupassen und gewisse Aspekte der Materie allenfalls im Gesetz selbst zu regeln. Soweit der St. Galler Gesetzgeber - in Kenntnis des angedachten Verordnungsrechts - dem Gesetzesentwurf der Regierung folgt und das Verordnungsrecht vom Regierungsrat dann tatsächlich auch wie vorgesehen erlassen wird, dürfte dies darauf hindeuten, dass sich das Verordnungsrecht an den Rahmen des Gesetzes hält. Dies gilt zumindest für die Zeit unmittelbar nach Erlass des betreffenden Gesetzes- und Verordnungsrechts. Je länger das gesetzgeberische Tätigwerden zurückliegt, desto weniger Gewicht kann der «impliziten Genehmigung» des Verordnungsrechts durch den Kantonsrat zukommen. Dies gilt jedenfalls dann, wenn sich das Gesetz, die Verordnung oder die Rahmenbedingungen im Laufe der Zeit verändert haben.

\section{5. $\quad$ Fazit}

[40] Mit der Überprüfung von Rechtsverordnungen überwacht die Judikative im Kern das Zusammenspiel und Spannungsverhältnis zwischen der Legislative und der Exekutive im Rahmen der Rechtssetzung. Insbesondere wenn zwischen dem Inkrafttreten einer gesetzlichen Delegationsnorm und dem Erlass oder der Revision einer gesetzesvertretenden Rechtsverordnung einige Zeit vergangen ist, besteht eine Tendenz der Gerichte, den ursprünglichen gesetzgeberischen Wil- 
len mit Blick auf ein befriedigendes Ergebnis der ratio legis zweckmässig zu interpretieren. Damit soll den inzwischen veränderten Rahmenbedingungen oder den Erkenntnissen aus der langjährigen Rechtsanwendung Rechnung getragen werden. Die Gerichte ergründen dabei, was der Gesetzgeber unter den mittlerweile veränderten Umständen gewollt hätte und ob die zu beurteilende gesetzesvertretende Rechtsverordnung vom gesetzgeberisch Gewollten (noch) erfasst wird. Dabei steht aber die Gefahr im Raum, dass die Gerichte den Willen des Gesetzgebers nachträglich in hypothetischer Weise anpassen oder erweitern. Bei einem solchen Vorgehen sind gewisse Rückschaufehler unausweichlich, und es besteht die Gefahr, dass die Grenze zwischen richterlicher Rechtsfindung und - unzulässiger - richterlicher Rechtsschöpfung überschritten wird. Das Bestreben, das Handeln der Exekutive und die Verordnungsnorm zu schützen, wirkt sich mithin nicht neutral aus, sondern hat unmittelbare Konsequenzen für das staatliche Machtgefüge. Unseres Erachtens sollten die Gerichte in solchen Konstellationen häufiger den Mut aufbringen, den Gesetzgeber anzuweisen, den veränderten Umständen, den Erkenntnissen aus der Rechtsanwendung oder dem gesellschaftlichen Wandel auf der Stufe des Gesetzes Rechnung zu tragen. Dafür ist jedoch notwendig, die Gesetzeswidrigkeit einer Verordnungsbestimmung ausdrücklich festzustellen, wenn die Verordnungsgeberin Regelungen ausserhalb des gesetzlich Delegierten aufstellt. Damit leisten die Gerichte im Zuge der Überprüfung von Rechtsverordnung einen wichtigen Beitrag zu einem effektiv gelebten Grundsatz der Gewaltenteilung.

Dr. iur. Arthur Brunner, Rechtsanwalt, Gerichtsschreiber der II. öffentlich-rechtlichen Abteilung des Schweizerischen Bundesgerichts, Lausanne, Ersatzmitglied am Verwaltungsgericht des Kantons Zürich, Lehrbeauftragter für öffentliches Recht an der Universität Zürich.

Dr. iur. Marco Zollinger, Rechtsanwalt, Gerichtsschreiber der II. öffentlich-rechtlichen Abteilung des Schweizerischen Bundesgerichts, Lausanne.

\section{Literaturverzeichnis}

Auer, Andreas (2016): Verfassungsgerichtsbarkeit, in: Auer, Andreas (Hrsg.), Staatsrecht der Schweizerischen Kantone, Bern, S. $591 \mathrm{ff}$.

Bachmann, Gregor (2019): Anspruch auf Verfahren und Entscheid, Diss. Freiburg.

Bolkensteyn, Arun (2014): Le contrôle des normes, spécialement par les cours constitutionnelles cantonales, Diss. Lausanne.

Brunner, Arthur (2020): COVID-19-Verordnung: Umstrittene Auslegung, NZZ Nr. 73 27.3.2020, S. 8.

Doleschal, Ralph David (2019): Die abstrakte Normenkontrolle in den Kantonen, Diss. Zürich.

Epiney, Astrid (2015): Art. 190 BV, in: Basler Kommentar Bundesverfassung, Basel, S. 2767 ff.

Graf, Martin (2014): Art. 151, in: Parlamentsrecht und Parlamentspraxis der Schweizerischen Bundesversammlung. Kommentar zum Parlamentsgesetz, Basel, S. 1020 ff.

Häfelin, Ulrich/Haller, Walter/Keller, Helen/Thurnherr, Daniela (2020): Schweizerisches Bundesstaatsrecht, 10. Auflage, Zürich/Basel/Genf.

Häfelin, Ulrich/Müller, Georg/Uhlmann, Felix (2020): Allgemeines Verwaltungsrecht, 8. Auflage, Zürich/St. Gallen.

Kelsen, Hans (2019): Wesen und Entwicklung der Staatsgerichtsbarkeit, in: Kelsen, Hans (Hrsg.), Wer soll der Hüter der Verfassung sein?, 2. Auflage, Tübingen, S. $1 \mathrm{ff}$.

Kocher, Martin (2018): Die bundesgerichtliche Kontrolle von Steuernormen. Grundlagen, Anfechtungsobjekt, Streitgegenstand, Verfahren, Fallstudien, Bern.

Müller, Georg (2020): Wie steuert der Gesetzgeber die Verordnung?, in: SJZ 116/2020 S. 47 ff.

Müller, Georg/Uhlmann, Felix (2013): Elemente einer Rechtssetzungslehre, 3. Auflage, Zürich/Basel/Genf. 
Nussbaum, Hans Georg (2003): Rahmenbedingungen der Verordnungsgebung: Rechtliche Grundlagen und Funktionen von Verordnungen, in: LeGes 14 (2003) 1, S. 9 ff.

Odermatt, Luzian/Tophinke, Esther (2014): Art. 22, in: Parlamentsrecht und Parlamentspraxis der Schweizerischen Bundesversammlung. Kommentar zum Parlamentsgesetz, Basel, S. $171 \mathrm{ff}$.

Schmid, Stefan (2018): Parlament und Regierung im Clinch: Das Verordnungsveto in seinen Grundzügen und im Lichte der Gewaltenteilung, in: ZBl 4/2018 S. $163 \mathrm{ff}$.

SChNeider, WAlter (1918): Die Verordnung im Rechtsstaat, Diss. Zürich.

Theler, Cornelia (2014): Art. 95, in: Parlamentsrecht und Parlamentspraxis der Schweizerischen Bundesversammlung. Kommentar zum Parlamentsgesetz, Basel, S. $671 \mathrm{ff}$.

Tschannen, Pierre/Zimmerli, Ulrich/Müller, Markus (2014): Allgemeines Verwaltungsrecht, 4. Auflage, Bern.

Uhlmann, Felix/Hofstetter, David (2012): Die Verordnung aus dem Blickwinkel der Rechtssetzungslehre, in: ZB1 113/2012 S. $455 \mathrm{ff}$.

Wyss, Martin (2020): Rechtsetzungsverfahren im Bund, in: Diggelmann, Oliver/Hertig Randall, Maya/Schindler, Benjamin (Hrsg.), Verfassungsrecht der Schweiz, Band III, Zürich/Basel/Genf, S. 1943 ff. 